\title{
Layout Optimization of Integrated Transfer Hub of Metropolis
}

\author{
Man Sun ${ }^{1, a}$, Shi $\mathrm{An}^{2, \mathrm{~b}}$ \\ ${ }^{1}$ School of Transportation Science and Engineering, Harbin Institute of Technology, 73 Huanghe \\ Road, Harbin, China, 150090 \\ ${ }^{2}$ School of Transportation Science and Engineering, Harbin Institute of Technology, 73 Huanghe \\ Road, Harbin, China, 150090 \\ asunman_hit@yahoo.com.cn, banshi@hit.edu.cn
}

\begin{abstract}
Key words: Integrated Transfer Hub; Demand Forecasting; Layout Optimization; Scheme Evaluation
\end{abstract}

\begin{abstract}
Integrated transfer hubs are the important transportation infrastructures and the passenger distributing center places, they play a very important role in promoting the development of transportation system. While domestic integrated transfer hubs exist some problems as not playing enough attention to the layout planning. The spatial layout and facility layout are reasonable and imperfect, what making low transfer efficiency, traffic bottlenecks and waste of resources. Therefore layout optimization of integrated transfer hub is a problem urgently to be solved.

Combine with features and running states of transit hubs in our country, passenger transport demand is forecasted and the combination forecasting model is constructed based on quadratic. In layout optimization of integrated transfer hub, the optimization model of facility layout is also put forward with minimum average cost as the object, making layout of hub effectively optimized. While proposing the optimization method, system evaluation are proposed aiming at the method, and internal benefits, social benefit and environmental benefit are used to evaluate the optimized system.
\end{abstract}

\section{Introduction}

Along with the fast development of our national economy, the transport industry in China entered a period of comprehensive progress. Building and improving our comprehensive transportation system becomes urgent need to develop the economy ${ }^{[1]}$. Comprehensive transport hub is a important constituent of a country or region's comprehensive transportation system. It involves in the transport industry in various technical processes, and it is the main base of various modes of transportation to converge. Integrated transfer hubs play an important role in promoting the function of comprehensive transport system, even in promoting the city development ${ }^{[2]}$. How to play a prominent role of integrated transfer hub in the city traffic network and regional integrated transport network effectively, to enhance the order of supply or demand, and to improve completeness of network structure, is the major problem that needs to solve urgently ${ }^{[3]}$.

Based on our country transportation hub station features and running states, we can find that, our country's integrated transfer hubs exist some problems as attaching importance on line construction while despising hub layout. Hub planning didn't get enough attention, what resulting in a distributed transfer and affecting the efficiency and benefit ${ }^{[4]}$. Unreasonable layout of interior hub made passengers walking very long distance to transit and resources utilized inadequately, so that to affect the efficiency of transportation system directly.

Foreign countries paid great attention to the study in construction of integrated transfer hubs. Research in hub plan, hub design and policy formulation had been carried out since 1950s. Many experiences and methods of hub design that fit city features were explored, which obtained good results $^{[5-6]}$. In recent years, in full recognition of the importance of transfer hub construction, domestic scholars had conducted many studies. Some metropolis constructed some integrated transfer hubs, such as Beijing South Railway Station Hub, Shanghai Hongqiao Hub and Shenzhen Futian Hub. 


\section{Influence factors and principles of integrated transfer hub layout}

\subsection{Influence factors of integrated transfer hub}

Integrated transfer hub layout planning is affected by many factors. It's an important step to analyze the layout factors for hub planning. The main factors can be summarized as follows.

(1)Social and economic factors

Social economic development impacts the hub design mainly in two aspects, the scale and the layout. In the scale of station, the more developed economic grown and more population in region, the more passenger demand generated. With the development of economy and the improvement of people's living standard, safety, convenience and other requirements have also been strengthened. In the layout of station, change of passenger demand and increase of travel frequency also affect the hub layout. Transfer hub layout should meet not only the downtown residents' needs, but also the suburbs' and the development zone residents ${ }^{\text {[7] }}$.

(2) Traffic conditions

(1) City road network condition. Different road network layout forms get different traffic characteristics. Layout of hub should give full consideration to the coordination and cohesion of city road network, so that to get smaller effects.

(2) External network connection. Integrated transfer hub design should plan station layout according to the different nature of road traffic, and get better convergence of the external road network. Making full use of existing resources can also reduce the impact on the city traffic.

(3) Modes of transportation development. Integrated transfer hub involves multiple transportation modes. Each different development traffic mode affects hub layout distinctly. Developed and mature traffic mode gets lager transfer passengers and assumes heavier transfer task, so it impacts the hub layout greater.

(4) Reachability of hubs. Different distribution of function area and traffic flow cause different time to achieve hub vary greatly. Reasonable hubs layout can reduce the transfer time of the passengers and the pressure on urban transport ${ }^{[8]}$.

(3) Urban layout factors

Development morphology and development function of cities affect nature of city, function region and development direction of cities. Each form has the own characteristics, which along with residents travel, economic activities and cultural activities determine the hub layout.

(4) Passenger travel factors

As passengers travel preferences and travel habits becoming increasingly important factor of the impact of layout, transfer hub meet needs of passengers at different levels and provide excellent transportation services is wise activity.

\subsection{Principles of integrated transfer hub}

Hub layout reasonable or not relate to the implementation of benefit and fulfilling of functions. Integrated hubs layout planning should follow the following principles.

(1)Meet the needs of regional transport development. Integrated hubs should not only meet the needs of the regional socio-economic and transport development planning, but also fully embodies the economic characteristics of the region to adapt to the needs of regional development ${ }^{[7]}$.

(2)Coordinate with the overall city layout planning. Spatial distribution structure and traffic flow should be given full consideration to. As the same time, future direction of urban development, resource distribution and population structure should also be considered to coordinate with other urban infrastructure and meet the needs of sustainable urban development.

(3)Coordinate with the development of road transport planning and integrated passenger transport network. Adapting to the road network development objective and matching with the main highway network can achieve the convenience of transport and give full play of advantages in the integrated transport.

(4)Make full use of the existing station resource and save the construction investment. On full study of the existing passenger transport hub, combined with the existing terminal condition, rational distribution can be conducted an resources can be made full use of. 
(5) Reduce the impact on the environment and the surrounding urban residents. Passengers and vehicles with frequent activities within hub would make on air pollution an noise pollution to the surrounding environment. Hub layout should consider the negative impact on the surrounding environment to reduce environmental interference.

\section{External transport passenger forecast}

There are many similarities between the hubs transfer amount forecast and traffic distribution forecast. Drawing on the traffic distribution $f\left(C_{i j}\right)$ forecast methods can help analyze passenger sharing. Construct an impedance function with the combination of power and exponent function, which choosing time, fee, comfort level and security level as the most significant factors to affect transfer behavior. There is the linear combination function ${ }^{[9]}$.

$C_{i}=C_{0}+k_{1 i} \cdot C_{1 i}+k_{2 i} \cdot C_{2 i}+k_{3 i} \cdot C_{3 i}+k_{4 i} \cdot C_{4 i}$

$C_{i} \longrightarrow$ generalized travel costs of transport mode i

$C_{1 i}, C_{2 i}, C_{3 i}, C_{4 i}$-transfer time, cost, comfort level, security level of transport mode $\mathrm{i}$

$C_{0} \longrightarrow$ other factors that influence transport $\mathrm{i}$

$k_{1 i}, \quad k_{2 i}, k_{3 i}, k_{4 i}$ corresponding coefficient

So the hub transfer passenger forecasting model is as follow:

$$
\begin{aligned}
& q_{i j}=a_{i} b_{j} O_{i} D_{j} f\left(C_{i j}\right) \\
& a_{i}=\frac{1}{\sum_{j} b_{i} D_{i}\left(C_{i j}\right)} \\
& b_{j}=\frac{1}{\sum_{i} b_{j} D_{j}\left(C_{i j}\right)} \\
& f\left(C_{i j}\right)=C_{i j}^{-\gamma} \cdot e^{-\beta C_{i j}}
\end{aligned}
$$

\section{Layout optimization of integrated transfer hub}

The goal to establish the transportation function layout mode in hub is to minimize the average transfer cost of all modes. There are some assumptions.

(1)There is one dominant mode, and subsidiary modes are $\mathrm{m} . \mathrm{k}=1,2, \ldots \mathrm{m}$.

(2)Alternative point of the subsidiary modes are $n$. $i=1,2, \ldots n$, and $n \geq m$

(3)Only one functional area which to construct proposed

(4)Alternative points get area restrictions ${ }^{[10]}$

Decision variables:

$X_{i k}= \begin{cases}1, & \text { alternative piont } i \text { is seleced as functional area } k \\ 0, & \text { alternative piont } i \text { is not seleced }\end{cases}$

The objective function mainly purpose to minimize the average transfer cost of all modes. Two parts need to be considered when calculating the total transfer cost. First, the transportation costs $C_{1}$ between the dominant mode and the subsidiary mode. Second, the transportation costs $C_{2}$ between subsidiary modes. $C_{0}$ is cost caused by other factors such as the construction cost-sharing and operation cost-sharing.

$\bar{C}=\frac{C}{N}=\frac{C_{1}+C_{2}+C_{0}}{N}$ 


$$
\begin{aligned}
& C_{1}=\sum_{i=1}^{n} \sum_{k=1}^{m}\left(N_{k}+N_{k}^{\prime}\right) X_{i k} \cdot c_{i} \\
& C_{2}=\sum_{i=1}^{n} \sum_{j=1}^{n} \sum_{k=1}^{m} \sum_{l=1}^{m} N_{k l} X_{i k} X_{j l} \cdot c_{i j} \\
& N=\sum_{k=1}^{m}\left(N_{k}+N_{k}^{\prime}\right)+\sum_{k=1}^{m} \sum_{l=1}^{m} N_{k l}
\end{aligned}
$$

The objective function:

$$
\min \bar{C}=\frac{\sum_{i=1}^{n} \sum_{k=1}^{m}\left(N_{k}+N_{k}^{\prime}\right) X_{i k} \cdot c_{i}+\sum_{i=1}^{n} \sum_{j=1}^{n} \sum_{k=1}^{m} \sum_{l=1}^{m} N_{k l} X_{i k} X_{j l} \cdot c_{i j}+C_{0}}{\sum_{k=1}^{m}\left(N_{k}+N_{k}^{\prime}\right)+\sum_{k=1}^{m} \sum_{l=1}^{m} N_{k l}}
$$

Constraints are as follows:

$$
\begin{aligned}
& \sum_{k=1}^{m} f\left(N_{k 0}\right) \cdot X_{i k} \leq S_{i} \quad i=1,2, \cdots n \\
& \sum_{k=1}^{m} X_{i k} \leq 1 \quad i=1,2, \cdots n \\
& \sum_{i=1}^{n} X_{i k}=1 \quad k=1,2, \cdots m \\
& \sum_{k=1}^{m} X_{i k} e_{k} \leq E \quad k=1,2, \cdots m
\end{aligned}
$$

$N_{k}$ — transfer amount from the dominant mode to subsidiary mode $\mathrm{k}$

$N_{k}^{\prime}$ — transfer amount from subsidiary mode $\mathrm{k}$ to the dominant mode

$N_{k l}$ — transfer amount from subsidiary mode $\mathrm{k}$ to $\mathrm{t}$ subsidiary mode 1

$N_{k 0} \longrightarrow$ total traffic flow of subsidiary mode $\mathrm{k}$

$c_{i} \longrightarrow$ generalized transfer cost from alternative site $\mathrm{i}$ to the dominant mode

$c_{i j} \quad$ generalized transfer cost from alternative site $\mathrm{i}$ to alternative site $\mathrm{j}$

$C_{0} \_$fixed cost sharing

$S_{i} \longrightarrow$ available area of alternative site i

$e_{k}-$ construction cost of transportation mode $\mathrm{k}$

E- total investment

In the mode: formula (4-5) is the objective function, meaning the minimum average transfer cost; formula (4-6) means meeting the area restriction when the alternative site is selected; formula (4-7) means each alternative site can only be constructed to one traffic functional region; formula (4-8) means each traffic functional region only choose one alternative site; formula (4-9) is the investment restriction.

The model above is nonlinear 0-1 integer programming model, which can be solved by heuristic algorithm. With the implicit enumeration in operations research and with computer programming, optimal solution in model can be obtained.

\section{Evaluation of integrated hubs layout}

(1) Internal benefit

Optimization layout of hub improve the level of convergence of the various modes of transportation and the transfer efficiency. It shortens transfer time, attract more passenger traffic and reduce the transfer cost. With the transfer time, transportation management fees, hub attracting capacity and traffic accident reduction as the indicators to measure the internal benefit. 


\section{(2) Social benefit}

Service range is expanded because of layout optimization, it makes the social benefits increasing combined with the urban transport system. Transfer hub contacts various modes of transportation, so that optimization of hub layout is the key to transportation modes convergence and external traffic distribution ${ }^{[11]}$. With the the congestion reduced efficiency, hub service range, degree of coordination with other hub and promotion of social development as the indicators to measure the social benefit.

(3) Environmental benefit

Layout optimization of urban external hubs can effectively reduce congestion, distribute traffic reasonably and reduce environmental pollution. It minimizes interference and impact on the surrounding environment and residents living. With the congestion reduction, environmental pollution effects, road service area reduction amount and the coordination degree with surrounding environment as the indicators to measure the environmental benefit.

\section{Conclusion}

Layout optimization of urban external integrated transport hub is a problem worthy of study. On the basis of passenger demand forecasting and distribution, the model was established with the minimum average transfer cost as the objective. Integrated transfer hub was optimized and evaluated from three aspects.

\section{References}

[1] Yuan Hong, Lu Huapu. Study on model and method of comprehensive transportation terminal. Journal Of Highway And Transportation Research And Development. 2001.03

[2] Peng Hui. Research on integrated transportation hub layout. Master degree thesis of Chang'an University. 2006

[3] Feng Wei. Theoretical Research on Key Problems of City's External Traffic Comprehensive Transfer Hubs System. Doctoral dissertation of Southwest Jiaotong University. 2010.

[4] Wang Jiancong. Key Issues on Transfer Organization of City Passenger Traffic Hubs. Doctoral dissertation of Beijing Jiaotong University.

[5] Marianov, V.and Serra, D.Loeation of hubs in a competitive environment. European Journal of Operational Resear c h, 114.1999, 3:63-71

[6] Mark C. Walkek. Planning and designing of on street light rail transit stations. Transportation Research Record 1361:3-9

[7] Zhou Li. The Study on Layout Method of Urban Highway Passenger Transport Hub. Master degree thesis of Beijing Jiaotong University. 2008

[8] Hu Along. Study on the Layout of Urban External Traffic Transfer Hubs. Master degree thesis of Southwest Jiaotong University. 2005.

[9] Luo Xia, Fang Dahong. Research on demand prediction of integrated transportation hub. Railway Transport and Economy.2009

[10] Jiang Lingyu. Study on Facility Layout Optimization of Integrated Passenger Transportation Hub. Master degree thesis of Southwest Jiaotong University. 2007

[11]Xie Tao. Study on Locating the Urban Transfer Terminals and Integrating the Traffic Resources. Master degree thesis of Dalian University of Technology. 2005. 